\title{
Regional Differences in Gamma-Aminobutyric Acid and Glutamate Concentrations in the Healthy Newborn Brain
}

\author{
(D) S.K. Basu, (D) S. Pradhan, (DS.D. Barnett, (D) M. Mikkelsen, (DK.J. Kapse, (D). Murnick, (D).L. Quistorff, \\ (D) C.A. Lopez, ID A.J. du Plessis, and (D)C. Limperopoulos
}

\begin{abstract}
BACKGROUND AND PURPOSE: Gamma-aminobutyric acid and glutamate system disruptions may underlie neonatal brain injury. However, in vivo investigations are challenged by the need for special ' $\mathrm{H}$-MR spectroscopy sequences for the reliable measurement of the neurotransmitters in this population. We used J-edited 'H-MR spectroscopy (Mescher-Garwood point-resolved spectroscopy) to quantify regional in vivo gamma-aminobutyric acid and glutamate concentrations during the early postnatal period in healthy neonates.
\end{abstract}

MATERIALS AND METHODS: We prospectively enrolled healthy neonates and acquired Mescher-Garwood point-resolved spectroscopy spectra on a 3T MR imaging scanner from voxels located in the cerebellum, the right basal ganglia, and the right frontal lobe. CSF-corrected metabolite concentrations were compared for regional variations and cross-sectional temporal trends with advancing age.

RESULTS: Fifty-eight neonates with acceptable spectra acquired at postmenstrual age of 39.1 (SD, 1.3) weeks were included for analysis. Gamma-aminobutyric acid (+ macromolecule) $(2.56$ [SD, 0.1]) i.u., glutamate (3.80 [SD, 0.2]), Cho, and mlns concentrations were highest in the cerebellum, whereas NAA (6.72 [SD, 0.2]), NAA/Cho, Cr/Cho, and Glx/Cho were highest in the basal ganglia. Frontal gamma-aminobutyric acid (1.63 [SD, 0.1]), Glx (4.33 [SD, 0.3]), $\mathrm{Cr}$ (3.64 [SD, 0.2]), and Cho concentrations were the lowest among the ROls. Glx, NAA, and Cr demonstrated a significant adjusted increase with postmenstrual age $(\beta=0.2-0.35)$, whereas gamma-aminobutyric acid and Cho did not.

CONCLUSIONS: We report normative regional variations and temporal trends of in vivo gamma-aminobutyric acid and glutamate concentrations reflecting the functional and maturational status of 3 distinct brain regions of the neonate. These measures will serve as important normative values to allow early detection of subtle neurometabolic alterations in high-risk neonates.

ABBREVIATIONS: J-DIFF = J-edited difference spectrum; GABA = gamma-aminobutyric acid; GABA+ = GABA with macromolecules; Glu = glutamate; GSH = glutathione; MEGA-PRESS = Mescher-Garwood point-resolved spectroscopy; NDI = neurodevelopmental impairment; NS = not significant; PMA = postmenstrual age; TEA $=$ term-equivalent age

G amma-aminobutyric acid (GABA) and glutamate (Glu) are principal neurotransmitters and play a critical role in fetal and neonatal brain development. ${ }^{1}$ Microstructural disruptions of neurotransmitter systems have been reported following preterm birth in animal and ex vivo human studies. ${ }^{2,3}$ Subtle disruptions may play a role in neurodevelopmental impairments (NDIs) observed in surviving premature infants even without structural

Received June 14, 2021; accepted after revision September 10

From the Department of Neonatology (S.K.B.), Developing Brain Institute (S.K.B., S.P., S.D.B., K.J.K., J.L.Q., C.A.L., C.L.), Division of Diagnostic Imaging and Radiology (C.L.), and Fetal Medicine Institute (A.J.d.P.), Children's National Hospital, Washington, DC; George Washington University School of Medicine (S.K.B., S.P. S.D.B., J.M., A.J.d.P., C.L.), Washington, DC; and Department of Radiology (M.M., J.M.), Weill Cornell Medicine, New York, New York.

The study was supported by grant funding from the National Institutes of HealthNational Heart, Lung, and Blood Institute award No. 1R01HL116585; Intellectual and Developmental Disabilities Research Center award No. 1U54HD090257; National Center for Advancing Translational Sciences award Nos. UL1TR001876 and KL2TR001877; and the A. James and Alice B. Clark Foundation award. Additional support was received from internal departmental funds of Children's National Hospital in Washington, DC. brain injury on conventional neuroimaging at term-equivalent age (TEA). ${ }^{4}$ Recent advances in ${ }^{1} \mathrm{H}$-MR spectroscopy, including $J$-edited Mescher-Garwood point-resolved spectroscopy (MEGAPRESS), have allowed detection of the otherwise overlapped GABA and glutamate signals. ${ }^{5,6}$ However, the application of these techniques to the neonate has been limited by technical challenges, including motion during nonsedated scans, suboptimal tissue segmentation and correction using adult algorithms, and a

The content is solely the responsibility of the authors and does not necessarily represent the official views of the Intellectual and Developmental Disabilities Research Center, the National Center for Advancing Translational Sciences, or the National Institutes of Health.

Please address correspondence to Catherine Limperopoulos, PhD, George Washington University School of Medicine, 111 Michigan Ave, NW, Washington, DC 20010; e-mail: climpero@childrensnational.org

- Indicates open access to non-subscribers at www.ajnr.org

Indicates article with online supplemental data.

http://dx.doi.org/10.3174/ajnr.A7336 


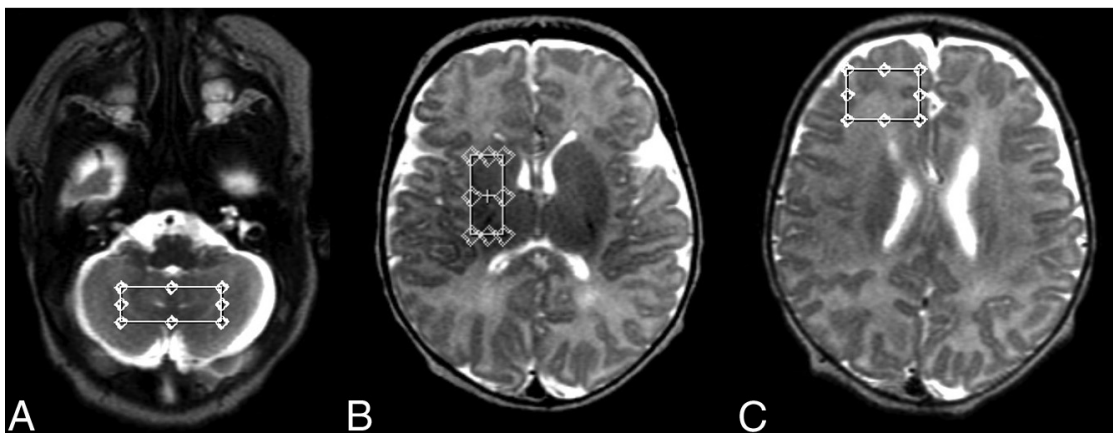

FIG 1. Voxel locations of ROls: cerebellum (A), right basal ganglia (B), and right frontal lobe (C).

\section{MATERIALS AND METHODS \\ Participants}

Healthy neonates born after 36 completed weeks of gestational age were prospectively enrolled between 2018 and 2020 in observational multimodal neuroimaging cohort studies. Neonates with known genetic syndromes, central nervous system anomalies, brain injury on MR imaging, or congenital infections were excluded. Clinical data were collected through medical record review and parental questionnaires. All studies were approved by the Children's

lower SNR from a limited voxel volume in the small neonatal brain, to name a few. ${ }^{7-11}$

Important knowledge gaps remain in our understanding of the normal temporal trajectory of in vivo GABA and glutamate concentrations during the critical perinatal transition and early postnatal maturation, which, in turn, limit the ability to detect abnormalities following premature birth. ${ }^{11}$ Animal and adult human studies have characterized the temporal trajectories of brain metabolites, their regional and sex-based variations, and the influence of mode of delivery; early postnatal studies, however, are currently limited. ${ }^{12-16}$ Conventional neonatal ${ }^{1} \mathrm{H}-\mathrm{MR}$ spectroscopy allows measurement of NAA and Cr (markers of neuronal integrity and metabolic activity), Cho (lipid membrane component), and mIns (cytoplasmic osmotic agent). Neonatal brain injury has been associated with lower NAA, ${ }^{17,18}$ and simultaneous GABA and glutamate measurements would allow in vivo neurotransmitter-specific interrogation of the developing brain. ${ }^{7-10,19}$

To fill these critical knowledge gaps, we measured in vivo GABA + and Glx concentrations from MEGA-PRESS spectra acquired from voxels placed in 3 ROIs: namely, the cerebellum, the right basal ganglia, and the right frontal lobe in healthy neonates. The cerebellum is known to play a regulatory-inhibitory role and accounts for most GABA and glutamatergic neurons in the human brain. The cerebellum matures rapidly during late gestation, with extended neurogenesis through infancy; and its parenchymal injury is associated with NDI in preterm infants. ${ }^{1}$ The basal ganglia are involved in sensorimotor organization and include an array of nuclei with glutamatergic and GABAergic neurons, and due to their high metabolic activity, they are vulnerable to the hypoxia-ischemia associated with premature birth. ${ }^{1}$ Finally, the frontal lobe is evolutionarily recent, mediates cognitive and executive function, but is relatively less mature in neonates. ${ }^{1}$

We hypothesized that these 3 critical brain ROIs would have distinct profiles of GABA+ and Glx as well as other metabolite concentrations in healthy neonates. We also hypothesized that NAA, Cr, and Glx would increase with advancing age (postmenstrual age [PMA]), whereas Cho (and $\mathrm{GABA}+$ ) would remain relatively stable. ${ }^{10,16,19,20}$ We sought to investigate the influence of postnatal age (weeks of life), sex, and mode of delivery on the neurometabolic profile of the neonatal brain.
National Hospital review board and conducted in accordance with relevant guidelines and regulations. Written informed consent was obtained from the parents of each study participant.

\section{MR Imaging and ' ${ }^{\mathbf{H}} \mathrm{H}$-MRS Acquisition}

Enrolled infants underwent a TEA MR imaging during natural sleep using the feed and swaddle technique on a 3T Discovery MR750 MR imaging scanner (GE Healthcare). Anatomic images were acquired using a T2-weighted 3D Cube (GE Healthcare; 3D FSE) sequence with the following parameters: section thickness = $1 \mathrm{~mm}$, section spacing $=0 \mathrm{~mm}, \mathrm{TR}=2500 \mathrm{~ms}$, TE $=64.7-$ $89.9 \mathrm{~ms}$, flip angle $=90^{\circ}$, orientation $=$ superior/inferior, number of slices $=120$, matrix $=160 \times 160 .{ }^{1} \mathrm{H}-\mathrm{MR}$ spectroscopy data were acquired from voxels placed in 3 ROIs: a $20 \times 15 \times 15 \mathrm{~mm}^{3}$ voxel centered on the white matter of the right frontal lobe, a $20 \times 20 \times 20 \mathrm{~mm}^{3}$ voxel centered on right basal ganglia, and a $25 \times 15 \times 10 \mathrm{~mm}^{3}$ voxel placed in the middle of the cerebellum (Fig 1). The MEGA-PRESS sequence was acquired using $\mathrm{TE}=$ $68 \mathrm{~ms}, \mathrm{TR}=2000 \mathrm{~ms}$, spectral width $=5000 \mathrm{~Hz}, 4096$ points, and 256 signal averages. Frequency-selective editing pulses (16-ms duration) were placed at $1.9 \mathrm{ppm}$ during $\mathrm{ON}$ and $7.5 \mathrm{ppm}$ during OFF acquisitions, respectively. J-edited difference (J-DIFF) spectra were generated by subtracting the OFF spectrum from the ON spectrum (composite J-DIFF and OFF spectra are shown in Fig 2) for each acquisition. Eight unsuppressed water averages were acquired for concentration referencing.

\section{'H-MR Spectroscopy Data Pre- and Postprocessing}

The pre- and postprocessing methods detailed previously ${ }^{7}$ included frequency and phase correction ${ }^{21}$ using the OFF and ON spectra to generate the J-DIFF spectrum. Each OFF and J-DIFF spectrum was analyzed using LCModel (http://www.lcmodel.com/) to measure metabolite concentrations using the unsuppressed water signal as an internal reference. ${ }^{22}$ The LCModel outputs were visually screened for artifacts, and only those with a full width at half maximum of $\leq 15 \mathrm{~Hz}$ and an SNR of $\geq 3$ for the OFF spectra and an SNR of $\geq 2$ for the DIFF spectra were included for further analysis.

GABA measurements from MEGA-PRESS spectra included contribution from coedited resonances from unspecified macromolecule resonances at $3 \mathrm{ppm}$ and hence are represented as $\mathrm{GABA}+{ }^{6}$ Analysis in the LCModel was performed using SPTYPE = MEGAPRESS-3 without inclusion of quantification of the macromolecule 

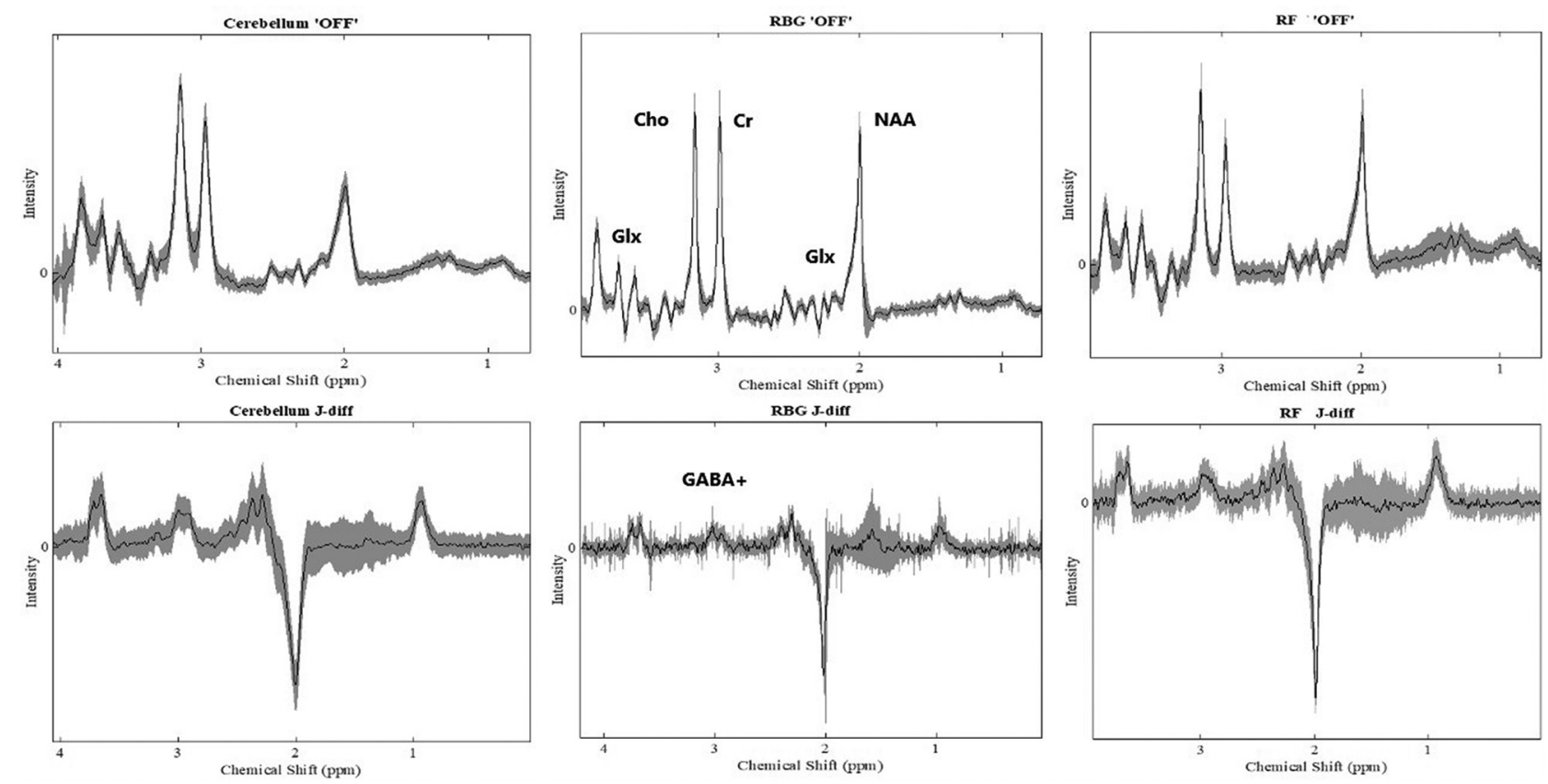

FIG 2. Composite 'H-MR spectroscopy PRESS OFF and J-DIFF spectra for all 3 ROls. Composite spectra from the 3 ROI voxels show the mean (solid line) and SD (shaded area) of all spectra included in the analysis. The spectra were acquired on a $3 \mathrm{~T}$ scanner with TE $=68 \mathrm{~ms}$, TR=2000 $\mathrm{ms}$, number of signal averages $=256$, and the editing pulses at 1.9 and $7.5 \mathrm{ppm}$. RBG indicates right basal ganglia; RF, right frontal lobe.

peak in the analysis. Hence, the GABA+ results reported correspond 1:1 to the GABA basis function scaled to the water peak.

Concentrations of Glu, glutamine, Glx, NAA, Cho, Cr, glutathione (GSH), and mIns were measured from the OFF spectra LCModel output. All metabolite concentrations are reported in international units. We accepted Cramer-Rao lower bound confidence intervals up to $50 \%$ for NAA, Cho, and Cr and up to $100 \%$ for other metabolites due to their lower concentrations, an a priori decision consistent with previous publications. ${ }^{7,19,23,24}$

\section{Tissue Segmentation and Correction for Voxel Composition}

The 3D T2 Cube images were automatically segmented using Draw-EM (https://github.com/MIRTK/DrawEM) and manually corrected in ITK-SNAP (www.itksnap.org) to measure global and regional brain volumes. The Gannet code (https://github. com/richardedden/Gannet3.1) with modifications to use segmented images exported from ITK-SNAP was used to generate voxel masks and was coregistered with segmented structural images to measure the voxel tissue fractions of CSF, GM, and $\mathrm{WM}^{25}$ Due to the intricate cerebellar folia, we segmented the cerebellar voxel into either GM + WM or CSF (ie, 2 tissue classes). Because intrinsic differences in metabolite levels between the GM and WM remain undetermined in the neonatal population, ${ }^{8}$ we used a composite GM + WM tissue fraction for CSF correction using the following equation:

$$
\text { Metabolite }_{\text {corrected }}=M_{\text {raw }} \div\left(f_{G M}+f_{W M}\right) \text {. }
$$

This approach assumes that there are negligible metabolite levels in the CSF and that GM and WM contribute equally to the metabolite signal in the spectra, ${ }^{8}$ effectively a CSF correction.

\section{Statistical Analysis}

Baseline characteristics of the cohort are presented as mean (SD) or frequency (percentage). Descriptive analyses of ${ }^{1} \mathrm{H}-\mathrm{MR}$ spectroscopy acquisition parameters and metabolite measurements, group (sex and mode of delivery), and regional comparisons were performed using nonparametric tests (ie, median, inter-quartile range, Wilcoxon Mann-Whitney test, and Kruskal-Wallis ANOVA, respectively). Spearman correlations were performed to investigate the relationships between metabolites and gestational age at birth, PMA, and postnatal age on the day of MR imaging. Linear mixed models were used to examine differences in metabolite concentrations among the 3 ROIs, adjusted for PMA. Post hoc ROI tests were conducted using contrasts. Potential confounding from clinical variables with previously reported $(\operatorname{sex})^{7}$ or biologically plausible (mode of delivery) ${ }^{11}$ associations with brain metabolites or neonatal outcomes was examined. Separate mixed-regression models were used to investigate the influence of PMA (model 1, adjusted for sex and mode of delivery) and postnatal age (model 2, adjusted for gestational age, sex, and mode of delivery) on metabolites on the day of MR imaging. Analyses were conducted using SAS 9.4 (SAS Institute). $P$ values $\leq .05$, two-tailed, were statistically significant. Sample size calculation and adjustment for multiple comparisons were not performed due to the descriptive nature of the study.

\section{RESULTS}

\section{Descriptive Characteristics of the Cohort}

Fifty-eight term control neonates born at a mean gestational age of 39.1 (SD, 1.3) weeks and birth weight of 3278 (SD, 464) g were included. Neonates underwent nonsedated MR imaging between 38.6 and 48.7 weeks' PMA, and their postnatal age ranged from 
0.7 to 8.9 weeks. Baseline characteristics of the cohort are detailed in Table 1.

\section{Voxel Characteristics}

The median voxel volume was largest for the cerebellum $\left(8.0 \mathrm{~cm}^{3}\right)$. All ROI voxels were composed $<10 \%$ by CSF proportion on average, with the highest gray matter proportion (99\%) in the right basal ganglia (Online Supplemental Data).

\section{Measured Raw and CSF-Corrected Metabolite Concentrations}

Of the 58 neonates included, 51 cerebellar, 36 right basal ganglia, and 48 right frontal spectra met quality inclusion criteria and raw (_r) metabolite concentration, and ratios are reported for them (Online Supplemental Data). We were able to determine tissue composition for 38/51 cerebellar, 30/36 basal ganglia, and 38/48 frontal lobe voxels and report CSF-corrected metabolite concentrations for them (Online Supplemental Data). Raw metabolite concentrations were not used for subsequent analyses (Tables 2 and 3). However, all metabolite ratios (from 51 cerebellar, 36

Table 1: Baseline clinical parameters $(n=58)^{a}$

\begin{tabular}{lc}
\hline \multicolumn{1}{c}{ Clinical Parameters } \\
\hline GA at birth (wk) & $39.1(\mathrm{SD}, 1.3)$ \\
Birth weight $(g)$ & $3278(\mathrm{SD}, 464)$ \\
Female sex & $28(48 \%)$ \\
Race & \\
African American & $8(15 \%)$ \\
White & $35(65 \%)$ \\
Others & $11(20 \%)$ \\
Singleton gestation & $60(100 \%)$ \\
Apgar score at 5 min (in median) & $9(9)$ \\
Vaginal delivery & $31(57 \%)$ \\
Maternal age (yr) & $35.4(\mathrm{SD}, 5.6)$ \\
PMA at MR imaging (wk) & $43.1(\mathrm{SD}, 2.4)$ \\
Postnatal age at MR imaging (wk) & $3.9(\mathrm{SD}, 2.1)$ \\
\hline
\end{tabular}

Note:-GA indicates gestational age.

${ }^{a}$ Data are means and frequency. (\%) unless otherwise noted. basal ganglia, and 48 frontal voxels) were included for regression analysis because CSF-correction factors cancel out for the ratios.

Adjusted for PMA (Table 2), cerebellar GABA+, Glu, glutamine, Cho, mIns, and GSH concentrations were the highest among the 3 ROIs, whereas NAA and Glx/Cho were the lowest. NAA concentrations and the ratio of NAA/Cho, $\mathrm{Cr} / \mathrm{Cho}$, and Glx/Cho were highest in the basal ganglia. The frontal lobe had the lowest GABA+, Glx, Cr, Cho, and GSH concentrations.

\section{Association of Sex, Race, and Mode of Delivery with Metabolite Concentrations}

Sex, race, and mode of delivery were not associated with significant differences in metabolite concentrations. Female neonates had lower right frontal lobe GABA+ (mean, 1.57 [SD, 0.4] i.u. versus $1.74[\mathrm{SD}, 0.5]$ ) i.u. concentrations compared with males, but they were not statistically significant (NS).

\section{Correlation of Metabolite Concentrations with Age}

Spearman correlations of GABA+ concentrations with PMA, gestational age at birth, and postnatal age did not demonstrate significant correlations (Spearman $\rho<0.2, P=\mathrm{NS}$ ), except for positive trends in the frontal lobe with PMA ( $\rho=0.34, P=.08$ ) and postnatal age $(\rho=0.35, P=.07)$ (Fig 3$)$. NAA, Cr, Glu, NAA/Cho, and $\mathrm{Cr} / \mathrm{Cho}$ positively correlated with PMA (Spearman $\rho=0.5-0.7, P<.01$ ) across all ROIs. Glx and Glx/ Cho correlated positively with PMA $(\rho=0.4-0.5, P<.01)$ and postnatal age $(\rho=0.3-0.4, P<.01)$ in the basal ganglia and the frontal voxels, but not in the cerebellum. Cho concentrations in the cerebellum and frontal lobe increased with PMA with a lower slope ( $\rho \sim 0.3-0.5, P<.03$ ), but not in the basal ganglia. Cho concentrations did not increase with advancing postnatal age $(<.3$, NS).

\section{Relationship between Brain Metabolites and PMA and Postnatal Age}

Multivariable regression analyses did not show any significant changes in GABA+ concentrations or ratios with age (Table 3). Basal ganglia Glx/Cho and NAA/Cho increased with PMA as well

Table 2: Regional difference in metabolite concentrations adjusted for PMA $^{\mathrm{a}}$

\begin{tabular}{|c|c|c|c|c|c|c|}
\hline & \multirow[b]{2}{*}{ Cerebellum } & \multirow[b]{2}{*}{ Right Basal Ganglia } & \multirow[b]{2}{*}{ Right Frontal Lobe } & \multicolumn{3}{|c|}{ Pair-Wise Comparisons ( $P$ Value) } \\
\hline & & & & C vs RBG & C vs RF & RBG vs RF \\
\hline \multicolumn{7}{|l|}{ Metabolites } \\
\hline GABA + & $2.56(\mathrm{SD}, 0.1)$ & $2.25(\mathrm{SD}, 0.1)$ & $1.63(\mathrm{SD}, 0.1)$ & $\uparrow .04$ & $\uparrow<.001$ & $\uparrow<.001$ \\
\hline Glx & $5.95(\mathrm{SD}, 0.2)$ & $5.68(\mathrm{SD}, 0.3)$ & $4.33(\mathrm{SD}, 0.3)$ & NS & $\uparrow<.001$ & $\uparrow<.001$ \\
\hline NAA & $3.84(\mathrm{SD}, 0.1)$ & $6.72(\mathrm{SD}, 0.2)$ & $4.31(\mathrm{SD}, 0.2)$ & $\downarrow<.001$ & $\downarrow .05$ & $\uparrow<.001$ \\
\hline $\mathrm{Cr}$ & $6.67(\mathrm{SD}, 0.1)$ & $6.72(\mathrm{SD}, 0.2)$ & $3.64(\mathrm{SD}, 0.2)$ & NS & $\uparrow<.001$ & $\uparrow<.001$ \\
\hline Cho & $3.08(S D, 0.1)$ & $2.33(\mathrm{SD}, 0.1)$ & $1.88(\mathrm{SD}, 0.1)$ & $\uparrow<.001$ & $\uparrow<.001$ & $\uparrow<.001$ \\
\hline Glu & $3.80(\mathrm{SD}, 0.2)$ & $3.78(\mathrm{SD}, 0.2)$ & 3.07 (SD, 0.2) & NS & $\uparrow .006$ & $\uparrow .02$ \\
\hline Gln & $2.55(\mathrm{SD}, 0.1)$ & $1.95(\mathrm{SD}, 0.2)$ & $1.72(\mathrm{SD}, 0.2)$ & $\uparrow .005$ & $\uparrow<.001$ & NS \\
\hline GSH & 1.89 (SD, 0.1) & $1.23(\mathrm{SD}, 0.1)$ & $1.05(\mathrm{SD}, 0.1)$ & $\uparrow<.001$ & $\uparrow<.001$ & NS \\
\hline mlns & $8.02(S D, 0.3)$ & $5.10(\mathrm{SD}, 0.3)$ & $6.05(S D, 0.3)$ & $\uparrow<.001$ & $\uparrow<.001$ & $\downarrow .017$ \\
\hline \multicolumn{7}{|c|}{ Metabolite ratios } \\
\hline $\mathrm{GABA}+/$ Cho & $0.85(\mathrm{SD}, 0.4)$ & $0.97(S D, 0.0)$ & $0.86(\mathrm{SD}, 0.4)$ & $\uparrow .03$ & NS & NS \\
\hline Glx/Cho & 1.97 (SD, 0.1) & $2.53(\mathrm{SD}, 0.1)$ & $2.26(\mathrm{SD}, 0.1)$ & $\downarrow<.001$ & $\downarrow .004$ & $\uparrow .026$ \\
\hline NAA/Cho & 1.31 (SD, 0.0) & $3.00(\mathrm{SD}, 0.1)$ & $2.34(\mathrm{SD}, 0.0)$ & $\downarrow<.001$ & $\downarrow<.001$ & $\uparrow<.001$ \\
\hline $\mathrm{Cr} / \mathrm{Cho}$ & $2.18(\mathrm{SD}, 0.0)$ & $2.96(\mathrm{SD}, 0.1)$ & $1.96(\mathrm{SD}, 0.0)$ & $\downarrow<.001$ & $\uparrow<.001$ & $\uparrow<.001$ \\
\hline $\mathrm{GABA}+/ \mathrm{Glx}$ & $0.45(\mathrm{SD}, 0.2)$ & $0.39(\mathrm{SD}, 0.0)$ & $0.41(\mathrm{SD}, 0.2)$ & NS & NS & NS \\
\hline
\end{tabular}

Note:- $\uparrow$ indicates higher than; $\downarrow$, lower than; C, cerebellum; RBG, right basal ganglia; RF, right frontal lobe; GLN, glutamine.

${ }^{a}$ Metabolites are marginal means (SD, standard error, in international units) adjusted for PMA. Metabolites are the following: $n=38$ for the cerebellum, $n=30$ for the right basal ganglia, and $n=38$ for the right frontal lobe. Metabolite ratios are the following: cerebellum $(n=51)$, right basal ganglia $(n=36)$, and left frontal lobe $(n=48)$. 


\begin{tabular}{|c|c|c|c|c|}
\hline & Age Parameter & Cerebellum ( $\beta, P$ Value) & $\begin{array}{l}\text { Right Basal Ganglia } \\
(\beta, P \text { Value })\end{array}$ & $\begin{array}{c}\text { Right Frontal Lobe } \\
(\beta, P \text { Value })\end{array}$ \\
\hline \multicolumn{5}{|l|}{ Metabolite } \\
\hline \multirow[t]{2}{*}{ GABA +} & Model 1': PMA (wk) & 0.02, NS & 0.02 , NS & $0.06, .11$ \\
\hline & Model $2^{\mathrm{c}}$ : postnatal age (wk) & -0.01, NS & $0.10, \mathrm{NS}$ & $0.09, \mathrm{NS}$ \\
\hline \multirow[t]{2}{*}{ Glx } & PMA & $0.25, .09$ & $0.22, .003$ & $0.3, .005$ \\
\hline & Postnatal age & $0.03, \mathrm{NS}$ & $0.15, .13$ & $0.14, \mathrm{NS}$ \\
\hline \multirow[t]{2}{*}{$\mathrm{Cr}$} & PMA & $0.29, .002$ & $0.11, .11$ & $0.20,<.001$ \\
\hline & Postnatal age & $0.15, \mathrm{NS}$ & 0.06, NS & $0.14, .06$ \\
\hline \multirow[t]{2}{*}{ Cho } & PMA & $0.06, .095$ & 0.02, NS & $0.05, .016$ \\
\hline & Postnatal age & $0.03, \mathrm{NS}$ & $0.01, \mathrm{NS}$ & $0.03, \mathrm{NS}$ \\
\hline \multirow[t]{2}{*}{ NAA } & PMA & $0.22, .002$ & $0.23,<.001$ & $0.36,<.001$ \\
\hline & Postnatal age & $0.14, .11$ & $0.17, .06$ & $0.27, .007$ \\
\hline \multicolumn{5}{|c|}{ Metabolite ratios } \\
\hline \multirow[t]{2}{*}{ Glx/Cho } & PMA & 0.02, NS & $0.10, .003$ & $0.09, .02$ \\
\hline & Postnatal age & $-0.02, \mathrm{NS}$ & $0.08, .038$ & $0.06, \mathrm{NS}$ \\
\hline \multirow[t]{2}{*}{$\mathrm{GABA}+/$ Cho } & PMA & $-0.01, \mathrm{NS}$ & $0.02, \mathrm{NS}$ & $0.02, \mathrm{NS}$ \\
\hline & Postnatal age & $-0.02, \mathrm{NS}$ & 0.02, NS & $0.03, .10$ \\
\hline \multirow[t]{2}{*}{ NAA Cho } & PMA & $0.05, .001$ & $0.13,<.001$ & $0.13,<.001$ \\
\hline & Postnatal age & $0.04, .04$ & $0.08, .002$ & $0.10,<.001$ \\
\hline \multirow[t]{2}{*}{$\mathrm{Cr} / \mathrm{Cho}$} & PMA & $0.05,<.001$ & $0.05, .12$ & $0.06,<.001$ \\
\hline & Postnatal age & $0.02, .14$ & $0.06, .13$ & $0.05, .015$ \\
\hline \multirow[t]{2}{*}{$\mathrm{GABA}+/ \mathrm{Glx}$} & PMA & $-0.02, \mathrm{NS}$ & $-0.02, .11$ & $-0.01, \mathrm{NS}$ \\
\hline & Postnatal age (wk) & $0.00, \mathrm{NS}$ & $-0.01, \mathrm{NS}$ & 0.00, NS \\
\hline
\end{tabular}

${ }^{a}$ Metabolites are the following: $n=38$ for the cerebellum, $n=30$ for the right basal ganglia, and $n=38$ for the right frontal lobe. Metabolite ratios were the following: cerebellum ( $n=51)$, right basal ganglia $(n=36)$, and left frontal lobe $(n=48)$.

${ }^{\mathrm{b}}$ Linear regression model 1: adjusted for PMA, sex, and mode of delivery.

'Linear regression model 2: adjusted for postnatal age, gestational age, sex, and mode of delivery.

GABA+

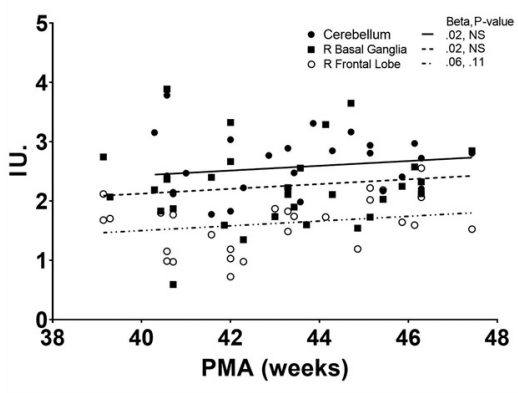

Glx

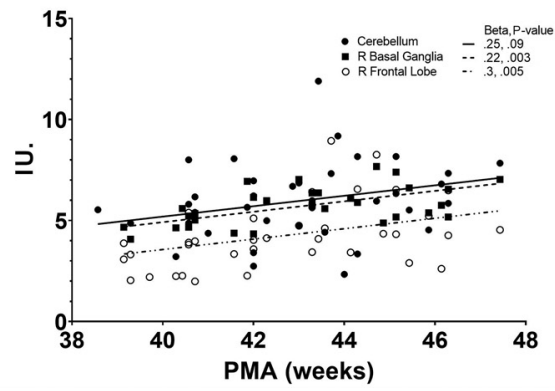

NAA

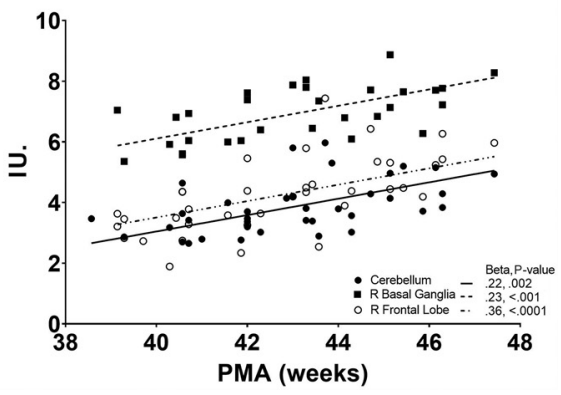

FIG 3. Temporal trajectories of metabolites with postmenstrual age. $R$ indicates right.

as postnatal age. Glx concentrations increased with PMA (NS for the cerebellum). NAA and NAA/Cho increased with PMA across all ROIs. Cho concentrations in the frontal lobe increased with PMA, but not with postnatal age in any of the ROIs.

\section{DISCUSSION}

We report, for the first time, normative data on regional in vivo variations of GABA and glutamate concentrations and their cross-sectional temporal trajectory during the early postnatal period. We observed distinct regional metabolic profiles in relationship to PMA and postnatal age, which may reflect differential maturational states and postnatal stimulation in the healthy neonate brain.

Our normative data from 3 ROIs of 58 healthy neonates are the largest cohort reported to date and will provide references for future investigations of alterations in neonates at risk of brain injury. The previous largest study, including 25 term neonates, reported higher right frontal GABA + concentrations compared with preterm neonates. ${ }^{9}$ Temporal increases in basal ganglia Glx and stable GABA+ concentrations during infancy were reported in preterm neonates, without data from term controls. ${ }^{19}$ One study including both term and preterm neonates reported higher GABA+ and Glx concentrations in the thalamus compared with anterior cingulate cortex, which lost statistical significance after tissue correction. ${ }^{8}$

We demonstrated that all metabolites except GABA+ positively correlated with advancing PMA, which likely reflects increasing metabolic activity of maturing neurons, dendrites, synapses, and glial cells (Fig 3). The stable GABA+ concentrations observed are consistent with those in previous animal ${ }^{12,16}$ and clinical studies in preterm infants. ${ }^{7,19}$ Consistent with previous studies, ${ }^{16,19}$ Cr concentrations increased significantly with PMA, whereas Cho remained relatively stable; hence, metabolite/Cho ratios are preferable markers of metabolic changes during early postnatal life. The duration of extrauterine exposure (postnatal 
age) demonstrated a significant positive correlation with basal ganglia Glx/Cho, perhaps indicating neuronal stimulation.

We found that NAA, NAA/Cho, Cr/Cho, and Glx/Cho (markers of neuronal integrity and metabolic activity) were highest in the basal ganglia, reflecting their relatively higher functional activity (Table 2). Also, the significant increase in $\mathrm{Cr}$ and $\mathrm{Glx} /$ Cho (NAA increase NS) within the basal ganglia with postnatal age (Table 3) indicates their metabolic and functional activity in response to extrauterine motor-sensory stimulation. Conversely, right frontal $\mathrm{GABA}+, \mathrm{Glx}$, and $\mathrm{Cr}$ concentrations were the lowest, consistent with their relative developmental immaturity in neonates. Cerebellar GABA+ and Glx concentrations were the highest, reflecting their dense neuronal composition. Cho, a marker of cell membrane turnover, was also highest in the cerebellum, perhaps inferring exuberant dendritic maturation as well as ongoing postnatal neurogenesis, as previously described in the human cerebellum. Glu, GSH (a cellular redox substrate), and mIns (cytoplasmic osmotic agent) were highest in the cell-dense cerebellum. The observed regional differences remained consistent for both raw and CSF-corrected metabolite concentrations. This finding may be due to the low CSF fraction of the voxels and that differences in gray-versus-white matter contributions to the metabolites could not be segregated.

Although not statistically significant, the lower right frontal GABA + concentration in female neonates (Table 3) agrees with findings in a recent report in preterm infants. ${ }^{7} \mathrm{We}$ did not observe any significant relationship of mode of delivery with regional metabolite concentrations, perhaps due to the delay in scan time (median, 4 weeks) after birth.

Although our study findings are strengthened by nonsedated scans in a modest size cohort of healthy neonates during early postnatal life, there are several limitations. The observed correlation of metabolite concentrations with PMA or postnatal age is cross-sectional and not longitudinal. Our tissue-correction strategy was limited to the CSF content of the voxel and assumed equal gray and white matter metabolite contributions. Neonatal algorithms and segmentation maps are needed for metabolite measurements adjusted for gray matter proportion from a neonatal brain voxel because adult algorithms may not reflect the many structural and maturational changes in the neonatal brain. Our data meet the quality parameters recommended in recent expert consensus statements, ${ }^{24,26,27}$ though one-fifth of the spectra had to be excluded due to motion or low SNR, inherent challenges with neonatal acquisitions. ${ }^{11}$

Regional differences in ${ }^{1} \mathrm{H}-\mathrm{MR}$ spectroscopy acquisition parameters like voxel size and SNR (Online Supplemental Data) may also influence these measurements, though if contributing, they would affect all metabolite measurements and would be nullified for metabolite ratios. The metabolite concentrations are expressed in international units due to their dependence on acquisition parameters and are not generalizable in terms of absolute concentrations across studies using different parameters. Interpretations need to consider that GABA+ has contributions from various macromolecules, whereas Glx has contributions from glutamine. Similarly, Glu, glutamine, and GSH measurements are not optimal from an unedited PRESS acquisition at $3 \mathrm{~T}$ due to poor spectral resolution, and adult studies have used higher magnetic field strengths, STEAM, or dedicated J-editing sequences. Despite these limitations, our study provides normative data and highlights regional differences and the influence of age on the metabolic milieu of the neonatal brain, which will aid in identification of alterations in disease states like neonatal encephalopathy or prematurity.

\section{CONCLUSIONS}

We report regional profiles of in vivo GABA+ and glutamate concentrations consistent with maturation and metabolic activity in the healthy neonatal brain. We also report increasing Glx, NAA, and Cr concentrations, but stable GABA and Cho concentrations during the neonatal period. The normative metabolic references may provide important, currently unavailable data that will allow detection of early metabolic and neurotransmitter alterations in neonates at high risk of NDI, even without significant structural brain injury.

\section{ACKNOWLEDGMENT}

We would like to acknowledge the contribution of the research nurses and assistants, MR imaging technicians, and other study staff whose relentless effort have made this study successful. Above all, we would like to thank all parents who have voluntarily allowed their children to undergo the MR imaging evaluations, without which this study would not have been possible.

Disclosure forms provided by the authors are available with the full text and PDF of this article at www.ajnr.org.

\section{REFERENCES}

1. Volpe JJ. Volpe's Neurology of the Newborn. Elsevier; 2018

2. Robinson S, Li Q, Dechant A, et al. Neonatal loss of gamma-aminobutyric acid pathway expression after human perinatal brain injury. J Neurosurg 2006;104(6 Suppl):396-408 CrossRef Medline

3. Shaw JC, Palliser HK, Dyson RM, et al. Disruptions to the cerebellar GABAergic system in juvenile guinea pigs following preterm birth. Int J Dev Neurosci 2018;65:1-10 CrossRef Medline

4. Laptook AR, O'Shea TM, Shankaran S, et al. Adverse neurodevelopmental outcomes among extremely low birth weight infants with a normal head ultrasound: prevalence and antecedents. Pediatrics 2005;115:673-80 CrossRef Medline

5. Harris AD, Saleh MG, Edden RA. Edited ${ }^{\mathbf{1}} \mathbf{H}$-magnetic resonance spectroscopy in vivo: methods and metabolites. Magn Reson Med 2017;77:1377-89 CrossRef Medline

6. Mullins PG, McGonigle DJ, O'Gorman RL, et al.; Cardiff Symposium on MRS of GABA. Current practice in the use of MEGA-PRESS spectroscopy for the detection of GABA. Neuroimage 2014;86:4352 CrossRef Medline

7. Basu SK, Pradhan S, Jacobs MB, et al. Age and sex influences gamma-aminobutyric acid concentrations in the developing brain of very premature infants. Sci Rep 2020;10:10549 CrossRef Medline

8. Maria YL, Price AN, Puts NA, et al. Simultaneous quantification of GABA, Glx and GSH in the neonatal human brain using magnetic resonance spectroscopy. Neuroimage 2021;233:117930 CrossRef Medline

9. Kwon SH, Scheinost D, Lacadie C, et al. GABA, resting-state connectivity and the developing brain. Neonatology 2014;106:149-55 CrossRef Medline 
10. Tomiyasu M, Aida N, Shibasaki J, et al. In vivo estimation of gamma-aminobutyric acid levels in the neonatal brain. NMR Biomed 2017;30:e3666 CrossRef Medline

11. Basu SK, Pradhan S, Du Plessis AJ, et al. GABA and glutamate in the preterm neonatal brain: in-vivo measurement by magnetic resonance spectroscopy. Neuroimage 2021;238:118215 CrossRef Medline

12. $\mathrm{Xu} \mathrm{G}$, Broadbelt $\mathrm{KG}$, Haynes $\mathrm{RL}$, et al. Late development of the GABAergic system in the human cerebral cortex and white matter. J Neuropathol Exp Neurol 2011;70:841-58 CrossRef Medline

13. Tkac I, Rao R, Georgieff MK, et al. Developmental and regional changes in the neurochemical profile of the rat brain determined by in vivo ${ }^{1}$ H NMR spectroscopy. Magn Reson Med 2003;50:24-32 CrossRef Medline

14. O'Gorman RL, Michels $\mathrm{L}$, Edden RA, et al. In vivo detection of GABA and glutamate with MEGA-PRESS: reproducibility and gender effects. J Magn Reson Imaging 2011;33:1262-67 CrossRef Medline

15. Castillo-Ruiz A, Mosley M, Jacobs AJ, et al. Birth delivery mode alters perinatal cell death in the mouse brain. Proc Natl Acad Sci U S A 2018;115:11826-31 CrossRef Medline

16. Ramu J, Konak T, Liachenko S. Magnetic resonance spectroscopic analysis of neurometabolite changes in the developing rat brain at 7T. Brain Res 2016;1651:114-20 CrossRef Medline

17. Koob M, Viola A, Le Fur Y, et al. Creatine, glutamine plus glutamate, and macromolecules are decreased in the central white matter of premature neonates around term. PLoS One 2016;11:e0160990 CrossRef Medline

18. Basu SK, Pradhan S, Kapse K, et al. Third trimester cerebellar metabolite concentrations are decreased in very premature infants with structural brain injury. Sci Rep 2019;9:1212-14 CrossRef Medline
19. Tanifuji S, Akasaka M, Kamei A, et al. Temporal brain metabolite changes in preterm infants with normal development. Brain Dev 2017;39:196-202 CrossRef Medline

20. Craddock RC, James GA, Holtzheimer PE, et al. A whole brain fMRI atlas generated via spatially constrained spectral clustering. Hum Brain Mapp 2012;33:1914-28 CrossRef Medline

21. Evangelou IE, Noeske R, Limperopoulos C. Retrospective correction of motion induced artifacts in $1 \mathrm{H}$ magnetic resonance spectroscopy of the fetal brain. In: Proceedings of the IEEE 12th International Symposium on Biomedical Imaging (ISBI), Brooklyn, New York, USA. April 16-19, 2015:853-57

22. Provencher SW. Automatic quantitation of localized in vivo $1 \mathrm{H} \mathrm{spec-}$ tra with LCModel. NMR Biomed 2001;14:260-64 CrossRef Medline

23. Kreis $\mathrm{R}$. The trouble with quality filtering based on relative CramerRao lower bounds. Magn Reson Med 2016;75:15-18 CrossRef Medline

24. Wilson M, Andronesi O, Barker PB, et al. Methodological consensus on clinical proton MRS of the brain: review and recommendations. Magn Reson Med 2019;82:527-50 CrossRef Medline

25. Edden RA, Puts NA, Harris AD, et al. Gannet: a batch-processing tool for the quantitative analysis of gamma-aminobutyric acid-edited MR spectroscopy spectra. J Magn Reson Imaging 2014;40:1445-52 CrossRef Medline

26. Lin A, Andronesi O, Bogner W, et al; Experts' Working Group on Reporting Standards for MR Spectroscopy. Minimum Reporting Standards for in vivo Magnetic Resonance Spectroscopy (MRSinMRS): experts' consensus recommendations. NMR Biomed 2021;34;e4484 CrossRef Medline

27. Choi IY, Andronesi OC, Barker P, et al. Spectral editing in ${ }^{1} \mathbf{H}-\mathbf{m a g}-$ netic resonance spectroscopy: experts' consensus recommendations. NMR Biomed 2021;34:e4411 CrossRef Medline 